\title{
Serological characterisation of Actinobacillus pleuropneumoniae isolated from pigs in 1993 to 1996
}

\author{
PJ BLACKALL, R BOWLES, JL PAHOFF and BN SMITH \\ Australasian Pig Institute, Queensland Department of Primary Industries, Animal Research Institute, Yeerongpilly Queensland 4105
}

Objectives To clarify the serological identity of the prototype strain of a group of Actinobacillus pleuropneumoniae isolates that could not be serotyped in previous studies and to establish the serovar of 378 isolates of $A$ pleuropneumoniae obtained from pigs in Australia over the period 1993 to 1996.

Design After initial validation, QGD and IHA tests were used to characterise the prototype isolate (HS143) selected to represent the cross-reacting isolates that were found in a previous study. Next, 378 recent field isolates of A pleuropneumoniae were characterised using the existing gel diffusion serotyping technique and/or the IHA or QGD tests.

Results The indirect haemagglutination test was shown to be capable of correctly recognising the reference strain for all serovars except serovar 11 . While the quantitative gel diffusion test was not as effective as indirect haemagglutination, it could recognise serovar 11 . When the two tests were used to examine the prototype strain (HS143) of the cross-reactive isolates, the results indicated that HS143 is serologically distinct from all 12 of the recognised serovars of $A$ pleuropneumoniae. However, as HS143 was subsequently identified as serovar 12 by one of the leading international reference laboratories, the antiserum to isolate HS143 was used as the serovar 12 antiserum. A total of 346 of the 378 A pleuropneumoniae field isolates examined could be confidently serotyped with almost $90 \%$ of the isolates being either serovar 1 (104 isolates); serovar 7 (83 isolates) or serovar 12 (142 isolates). A range of other serovars and some cross-reactive isolates made up the remainder of the isolates.

Conclusion The serovar 12 antiserum produced against the international reference strain (1096) does not recognise Australian serovar 12 isolates. The antiserum raised against isolate HS143 does recognise the Australian serovar 12 isolates. The dominant serovars of $A$ pleuropneumoniae infecting Australian pigs are (in decreasing order) serovars 12 , 1 and 7.

Aust Vet J 1998;76:39-43

Key words: Actinobacillus pleuropneumoniae, serotyping, pig.

$A$ ctinobacillus pleuropneumoniae is the causative agent of porcine pleuropneumonia, a severe respiratory disease of pigs that is of economic importance where-ever pigs are raised. ${ }^{1}$ Twelve serovars of this important bacterial pathogen are currently recognised. ${ }^{1} \mathrm{~A}$ knowledge of the serovars prevalent in a region or on a farm is important as inactivated vaccines can protect only against those serovars in the vaccine.,2,3

A range of methods has been reported for the serotyping of $A$ pleuropneumoniae. ${ }^{4}$ The methods that have found widespread use are rapid slide agglutination, GD, IHA, ring precipitation, coagglutination and counterimmunoelectrophoresis. ${ }^{4-8}$ None of these techniques is perfect - cross-reactions, particularly between serovars 1,9 and 11 and between serovars 3, 6 and 8, are a problem with most of these techniques. ${ }^{9,10}$
In the most recent study on the serological characterisation of Australian isolates of $A$ pleuropneumoniae, a large percentage of isolates either gave cross-reactions with serovars 3 and 6 (47/256 isolates) or no reaction at all (91/256 isolates). ${ }^{11}$ This study was performed using slide agglutination and GD, techniques which had performed effectively in the past. ${ }^{5}$ The high incidence of isolates that could not be clearly serotyped was clear evidence that these methods were no longer as effective.

Several recent studies have shown that, of the available serotyping methods, IHA and QGD are the most effective methods for correctly serotyping A pleuropneumoniae isolates. ${ }^{10,12,13}$ In this paper, we describe the set-up and validation of IHA and QGD for the serotyping of A pleuropneumoniae using the reference strains for the 12 serovars and rabbit hyper-immune sera raised against these 12 strains. The use of the IHA and QGD to examine isolate HS143, selected to represent the problem isolates from the earlier study ${ }^{11}$ is then reported. In addition, we describe the serological characterisation of 378 isolates of $A$ pleuropneumoniae obtained as part of a national referral service for the identification of porcine haemophili. We also re-examine a subset of the cross-reacting and non-typable isolates reported in our previous serotyping study. ${ }^{11}$

\section{Materials and methods \\ Bacteria}

The 12 reference strains for the recognised serovars of $A$ pleuropneumoniae (strains 4074, 4226, 1421, M62, K17, Femo, WF83, 405, CVJ 13621, 22009, 56153 and 1096, serovars 1 to 12 respectively) were used. Isolate HS143 was obtained in 1990 from a breeding herd within Company A. The 378 field isolates of A pleuropneumoniae were obtained from veterinary diagnostic laboratories in New South Wales (68 isolates), Queensland (117 isolates), South Australia (20 isolates), Victoria (157 isolates) and Western Australia (16 isolates). All 378 isolates were shown to be A pleuropneumoniae using the phenotypic testing methods previously described. ${ }^{11}$

\section{Antisera}

The antisera for serovars 1 to 12 were those used in previous studies. ${ }^{5,11}$ Antiserum against isolate HS143 was produced as described previously for the reference strains. ${ }^{5}$

$\begin{array}{ll}\text { GD } & \text { Gel diffusion } \\ \text { IHA } & \text { Indirect haemagglutination } \\ \text { NA } & \text { Noble agar, Difco } \\ \text { PBS } & \text { Phosphate_buffered saline } \\ \text { QGD } & \text { Quantitative gel diffusion } \\ \text { SRBCs } & \text { Sheep red blood cells }\end{array}$




\section{Serotyping methods}

The bacteria were grown on Test Medium Agar supplemented with chicken serum and nicotinamide adenine dinucleotide, termed TM/SN agar, ${ }^{14}$ at $37^{\circ} \mathrm{C}$ for 5 h. The growth was then harvested into $2 \mathrm{~mL}$ of $0.15 \mathrm{M} \mathrm{NaCl}$. The suspension was washed and resuspended in PBS ( $\mathrm{pH}$ 7.2) containing thiomersal $(0.01 \%$ final volume). The antigen was held for $30 \mathrm{~min}$ at $56^{\circ} \mathrm{C}$ and then centrifuged $\left(11,000 \mathrm{~g}, 4^{\circ} \mathrm{C}, 30 \mathrm{~min}\right)$ and the supernatant retained for use in the various serological tests.

The GD test was performed in a medium that consisted of $1 \%$ NA in PBS containing $3 \mathrm{mM}$ sodium azide. For the QGD test, each antigen was tested against each serum over the following serum dilution range: $1 / 2,1 / 4,1 / 5,1 / 10,1 / 20,1 / 40$ and $1 / 80$.

The IHA test was performed using a method provided by Dr R Nielsen (State Veterinary Serum Laboratory, Copenhagen, Denmark). Briefly, the antigen was diluted 1:10 in PBS. SRBCs were collected into an equal volume of Alsiever's solution. The SRBCs were washed three times in PBS and resuspended to $4 \%$ in PBS. The SRBCs were coated with diluted antigen by mixing equal volumes of the antigen and $4 \%$ SRBCs and holding the mixture at $37^{\circ} \mathrm{C}$ for $90 \mathrm{~min}$. The sensitised SRBCs were washed three times in PBS and resuspended to a final concentration of $1 \%$. The antisera were heat inactivated $\left(56^{\circ} \mathrm{C}, 30 \mathrm{~min}\right)$ prior to use and non-specific agglutinins removed by absorption with four volumes of $4 \%$ SRBCs $\left(37^{\circ} \mathrm{C}, 10 \mathrm{~min}\right)$. Fifty $\mu \mathrm{L}$ of sensitised SRBCs and $50 \mu \mathrm{L}$ of diluted antisera were used in the actual tests, which were performed in U-bottom microtitre trays. The plates were incubated at room temperature for $2 \mathrm{~h}$. The sera were tested as doubling dilutions from $1 / 5$ to $1 / 10,240$.

For the field isolates, serotyping was initially performed using the GD test. For those isolates that gave a single reaction, this was accepted as the serovar. If isolates were nontypable or reacted with more than one antiserum in the GD test, they were then tested with the IHA test. Isolates were assigned to the serovar for which the highest titre was recorded. For those isolates that gave equal titres with several serovars, the QGD test was then used. The result from the QGD test was accepted as the definitive result for the isolate.

A subset of 74 isolates from the previous 1988-1992 serotyping study ${ }^{11}$ were re-examined using the HS143 antiserum and the GD and/or IHA tests.

\section{Results}

The results of testing all 12 reference antisera against all 12 reference antigens by QGD are shown in Table 1 . For 10 of the 12 reference antigens, only the expected homologous reactions were detected. With the serovar 9 antigen, a low titre of $1 / 2$ was detected against serovars 3 and 9. For the serovar 11 antigen, a cross-reaction was detected against serovar $9(1 / 2)$ although this was lower than the homologous serovar 11 titre $(1 / 4)$.

The results of testing all 12 reference antisera against all 12 reference antigens by IHA are shown in Table 2. Ten of the 12 reference antigens gave only the expected homologous reaction. With the serovar 8 antigen, a cross-reaction with serovar 6 was detected, although this heterologous reaction was a lower titre $(1 / 80)$ compared with the homologous titre (1/320). With the serovar 11 antigen, the antisera for serovars 9 and 11 gave equal titres $(1 / 2560)$

The results obtained with testing HS143 antigen against all 12 reference antisera as well as all 12 antigens against the HS143 antiserum by QGD and IHA are presented in Tables 1 and 2 respectively. By IHA, HS143 appears to be serologically distinct. The HS143 antigen reacted only with the HS143 antiserum. As well, none of the 12 reference antigens reacted with the HS143 antiserum. The results with the QGD test were not as clear cut. The HS143 antigen gave low titres with the serovar 9 and 11 antisera, $1 / 2$ and $1 / 4$ respectively. A titre of $1 / 4$ was found using the HS143 antigen and the HS143 antiserum.

In order to confirm the IHA results with HS143, which strongly suggested that this isolate represents a new serovar, this isolate was examined in the laboratory of Dr Nielsen (State Veterinary Serum Laboratory, Denmark). When tested in Denmark, isolate HS143 was shown to react with the Danish serovar 12 antiserum with a titre of $1 / 160$ in a IHA test (PJ Blackall unpublished data). As the laboratory of Dr Nielsen is the internationally recognised centre for the serotyping of $A$ pleuropneumoniae, HS143 has been accepted as serovar 12. Any Australian field isolate reacting specifically with the HS143 antiserum can be identified as serovar 12 .

The serotyping of the 378 field isolates was performed using antisera raised against the international reference strains for serovars 1 to 11 plus the HS143 antiserum as the serovar 12 antiserum. The serovars of the 378 isolates of $A$ pleuropneumoniae are shown in Table 3, which presents the combined results for the three serotyping methods used in this study - GD, IHA

Table 1. Results of cross-serovar testing using the quantitative gel diffusion test.

\begin{tabular}{|c|c|c|c|c|c|c|c|c|c|c|c|c|c|}
\hline \multirow[t]{2}{*}{ Antigen } & \multicolumn{13}{|c|}{ Antiserum } \\
\hline & 1 & 2 & 3 & 4 & 5 & 6 & 7 & 8 & 9 & 10 & 11 & 12 & 143 \\
\hline 1 & 20 & - & - & - & - & - & - & - & - & - & - & - & - \\
\hline 2 & - & 40 & - & - & - & - & - & - & _ & - & - & - & - \\
\hline 3 & - & - & 2 & - & - & - & - & - & - & - & - & - & - \\
\hline 4 & - & - & - & 10 & - & - & - & - & - & - & - & - & - \\
\hline 5 & - & - & - & - & 20 & - & - & - & - & & - & - & - \\
\hline 6 & - & - & - & - & - & 20 & - & - & - & - & - & - & - \\
\hline 7 & - & - & - & - & - & - & 20 & - & - & - & - & - & - \\
\hline 8 & - & - & - & - & - & - & - & 40 & - & - & - & - & - \\
\hline 9 & - & - & 2 & - & - & - & - & - & 2 & - & - & - & - \\
\hline 10 & - & - & - & - & - & - & - & - & - & 5 & - & - & - \\
\hline 11 & - & - & - & - & - & - & - & - & 2 & - & 4 & - & - \\
\hline 12 & - & - & - & - & - & - & - & - & - & - & - & 10 & - \\
\hline 143 & - & - & - & - & - & - & - & - & 2 & - & 4 & - & 4 \\
\hline
\end{tabular}

Results are expressed as the inverse of the antiserum titre. 
Table 2. Results of cross-serovar testing using the indirect haemagglutinin test.

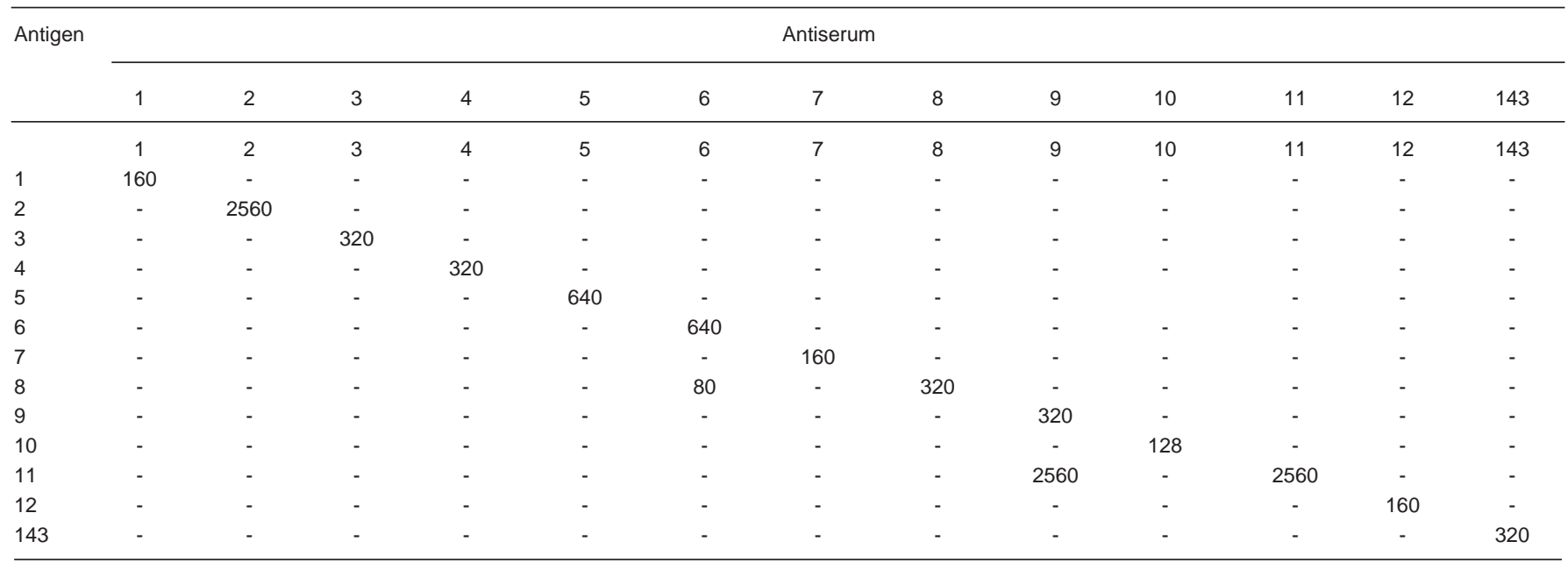

Results are expressed as the inverse of the antiserum titre.

Table 3. Results of serotyping of $\mathbf{3 7 8}$ Australian isolates of

A pleuropneumoniae.

\begin{tabular}{lccccccc}
\hline Serovar & NSW & QLD & SA & VIC & WA & Total & Percentage \\
\hline 1 & 12 & 0 & 3 & 83 & 6 & 104 & 27.5 \\
2 & 1 & 3 & 0 & 0 & 1 & 5 & 1.3 \\
3 & 0 & 2 & 1 & 0 & 0 & 3 & 0.8 \\
5 & 0 & 8 & 0 & 1 & 0 & 9 & 2.4 \\
7 & 16 & 32 & 8 & 23 & 4 & 83 & 22.0 \\
12 & 38 & 58 & 1 & 42 & 3 & 142 & 37.6 \\
NT & 0 & 5 & 0 & 6 & 2 & 13 & 3.4 \\
2,5 & 0 & 1 & 0 & 0 & 0 & 1 & 0.3 \\
$2,10,11$ & 0 & 1 & 0 & 0 & 0 & 1 & 0.3 \\
3,12 & 0 & 2 & 0 & 0 & 0 & 2 & 0.5 \\
$3,6,10,12$ & 0 & 2 & 1 & 0 & 0 & 3 & 0.8 \\
$3,7,10$ & 1 & 0 & 0 & 0 & 0 & 1 & 0.3 \\
7,10 & 0 & 2 & 2 & 1 & 0 & 5 & 1.3 \\
7,12 & 0 & 1 & 0 & 0 & 0 & 1 & 0.3 \\
10,12 & 0 & 0 & 4 & 1 & 0 & 5 & 1.3 \\
TOTAL & 68 & 117 & 20 & 157 & 16 & 378 & 100 \\
\hline
\end{tabular}

and QGD. About $91 \%$ of the isolates (346/378) were allocated to single serovars. Of the isolates that were allocated to a serovar, 329 (87\% of the total) were allocated to just three serovars: 1,7 and 12 . The most common serovars were serovar 12 (142 isolates) followed by serovar 1 ( 104 isolates) followed by serovar 7 (83 isolates). No other serovar contained over nine isolates. Only a small percentage of the isolates were either non-typable $(3.4 \%)$ or gave cross-reactions with a number of serovars $(5.1 \%)$.

Isolates of serovars 7 and 12 were derived from all five States represented in this study. A notable feature was the absence of serovar 1 in Queensland. As the total number of isolates of $A$ pleuropneumoniae from Queensland was large (117 out of the 378 examined), the absence of serovar 1 was not coincidental. Apart from Queensland, serovar 1 was present in all States covered in this study. The remaining serovars, 2, 3 and 5, were found in small numbers only and had limited distribution.

The results of the re-examination of a subset of isolates from the previous serotyping study ${ }^{11}$ resulted in the 74 isolates, previ- ously typed as either serovar 3,6 crossreacting (29 isolates) or non-typable (45 isolates), being identified as serovar 12 (64 isolates) or non-typable (10 isolates).

\section{Discussion}

The validation results obtained with the IHA serotyping method indicated that this is a very effective method of serotyping A pleuropneumoniae. Of the 12 reference strains examined, 10 gave only the expected homologous reactions. The cross-reaction detected between serovars 6 and 8 has been noted by others. ${ }^{9,15,16}$ The difference in titres between the homologous and heterologous reactions is such that correct allocation to serovar 6 or 8 is easily achieved. The IHA method could not distinguish between serovars 9 and 11, a finding that has been reported previously. ${ }^{12}$

The QGD serotyping method was as effective as the IHA, although titres were much lower. In the QGD method, 10 of the 12 reference strains gave only the expected homologous reactions. Unexpectedly, the QGD method, when used on the serovar 9 antigen, gave equal titres to serovars 3 and 9 antiserum. However, the QGD method did correctly serotype the serovar 11 strain, although a cross-reaction to serovar 9 was seen.

Overall, we found that both the IHA and QGD methods were effective and were able to clearly differentiate at least 10 of the 12 recognised serovars without any evidence of the crossreactions that can cause problems in the other methods. ${ }^{9,15-17}$ As neither method could recognise all 12 serovars, it would seem that a combination of both methods may have to be used for definitive serotyping.

The IHA results obtained in Australia with HS143 strongly suggest that this organism represents a new serovar. However, in a collaborative study performed in the laboratory of Dr Nielsen in Denmark, isolate HS143, using both antigen produced in Australia and Denmark, was shown to react with serovar 12 with a titre of 1/160 in an IHA test (PJ Blackall unpublished data). Further, the serovar 12 reference antiserum produced in Australia was shown to have a much lower titre to the reference serovar 12 strain (1096) than the equivalent antiserum produced in Denmark in the IHA test (1/160 compared with 1/2560) (PJ Blackall unpublished data). Hence, it appears that 
the reason the Australian-produced serovar 12 reference serum failed to type HS143 as serovar 12 is due to the fact that the Australian antiserum is weaker and has a lower homologous titre. Based on the results obtained in the laboratory of Dr Nielsen, HS143 antiserum can be used as a substitute serovar 12 antiserum. Field isolates reacting specifically with the HS143 antiserum can be identified as serovar 12 .

An alternative hypothesis on the nature of the isolates that have been shown to react with the HS143 antiserum is that these isolates are not serovar 12 but rather represent a new serovar. For this hypothesis to be accepted as correct, the findings of the serological identity of HS143 as serovar 12 by Dr Nielsen would have to be ignored. There is no accepted, definitive 'reference' laboratory that can provide a guaranteed 'correct' answer for the serotyping of $A$ pleuropneumoniae. However, the fact that 4 of the currently recognised 12 serovars, including serovar 12 itself, were proposed by Dr Nielsen ${ }^{1}$ makes the finding by Dr Nielsen that HS143 is serovar 12 as definitive an answer as is currently possible.

A notable result of the serotyping of A pleuropneumoniae field isolates was the predominance of serovars 1,7 and $12-87 \%$ of the 378 isolates belonging to just these three serovars. While serovars 1 and 7 have been dominant serovars in the previous two studies, ${ }^{5,11}$ serovar 12 has not been recognised as a dominant serovar previously. Table 4 shows the predominant serovars found in all three serotyping studies that have been completed to date. A major difference between the results of the current study and those of the previous studies has been the emergence of serovar 12. Our re-examination of a subset of the nontypable isolates from the earlier serotyping 1988-1992 study ${ }^{11}$ established that over $85 \%$ of the problem isolates examined were, in fact, serovar 12 . Hence, the emergence of serovar 12 began over the term of the 1988-1992 study. ${ }^{11}$ However, the emergence was not recognised due to the technical failings of the sera and the antigen extraction techniques used in that study. As the methods used in the first study ${ }^{5}$ were the same as the 1988-1992 study ${ }^{11}$ and there were few non-typable or crossreacting isolates in the first study, it can be concluded that the 'problem' or serovar 12 isolates were only detected after 1987.

The current study has shown that, at the national level, the relative prevalence of serovar 1 has decreased (47\% in 1988 to $27.5 \%$ in 1996) and that of serovar 12 has greatly increased ( $0 \%$ in 1988 to $37.6 \%$ in 1996). The emergence of serovar 12 is mostly explained by the addition of the IHA and QGD tests as serotyping methods, the altered antigen preparation method compared with that used in the earlier studies ${ }^{5,11}$ and the recognition that isolates reacting with the HS143 antiserum were serovar 12 (allowing many isolates to be assigned to that serovar). One other possible factor contributing to the emergence of serovar 12 is the fact that many more diagnostic laboratories are now examining samples from pig lungs as a result of the widespread adoption of pig health monitoring schemes. We have already reported that the so-called serovar 3,6 crossreacting isolates (now known to be serovar 12) are more frequently obtained from slaughter checks than isolates of serovar $1 .{ }^{11} \mathrm{It}$ is possible that the specific monitoring of pigs at slaughter for pneumonia and pleurisy resulted in greater efforts to isolate the cause of pneumonic lesions, resulting in the increase of serovar 12 isolates. Yet another factor is that serovar 12 may have become widely disseminated throughout the Australian pig herd by the presence of this serovar in a breeding pyramid.
Table 4. Comparison of the three A pleuropneumoniae serotyping studies performed to date.

\begin{tabular}{lccc}
\hline \multirow{2}{*}{ Serovar } & \multicolumn{3}{c}{ Percentage of isolates } \\
\cline { 2 - 4 } & Eaves and Blackall $^{5}$ & Blackall and Pahoff $^{11}$ & Current study \\
\hline 1 & 47 & 28.7 & 27.5 \\
7 & 29 & 17.2 & 22 \\
12 & 0 & 0.7 & 37.6 \\
Non-typable & 2 & 47.3 & 8.5 \\
\hline
\end{tabular}

Table 5. Relative prevalence of serovars 1, 7 and 12 of $A$ pleuropneumoniae in Queensland, New South Wales and Victoria.

\begin{tabular}{lcccc}
\hline \multirow{2}{*}{ Serovar } & \multicolumn{4}{c}{ Percentage of isolates } \\
\cline { 2 - 5 } & New South Wales & Queensland & Victoria & National \\
\hline 1 & 17.6 & 0 & 52.9 & 27.5 \\
7 & 23.5 & 27.3 & 14.7 & 22 \\
12 & 55.9 & 49.6 & 26.7 & 37.6 \\
\hline
\end{tabular}

While the overall prevalence figures indicate that the dominant serovars are (in decreasing order) serovar 12,1 and 7, it is important to note that this prevalence is not universal. Three States (New South Wales, Queensland and Victoria) provided enough isolates to enable a meaningful examination of the prevalence of serovars in those States (Table 5). None of the three States showed the same percentage distribution of these three serovars as the overall national figures. A particular feature is the absence of serovar 1 from Queensland isolates, despite the fact that this State contributes the second largest number of isolates overall.

These findings of regional variation in serovar prevalence combined with the dramatic shift in serovar prevalence over the time period from the first of our studies 5 highlights the need for continued serotyping studies.

Our finding that almost $90 \%$ of Australian A pleuropneumoniae isolates belong to only three serovars is of considerable practical importance. Inactivated pleuropneumonia vaccines, the only currently available vaccines for this disease, protect only against those serovars present in the vaccine. ${ }^{2,3}$ Hence, our findings strongly suggest that inactivated pleuropneumonia vaccines for use in Australia should contain serovars 1, 7 and 12. As other serovars are present (notably serovars 2, 3 and 5), isolates of A pleuropneumoniae associated with apparent vaccine failures should be serotyped. Alternatively, veterinarians considering use of an inactivated vaccine in a particular herd could have the serovar of A pleuropneumoniae responsible for the disease established to confirm that the proposed vaccine contains the correct serovar(s).

A possible consequence of the use of inactivated vaccines containing the predominant serovars (1, 7 and 12) is a 'drift' away from those serovars in the vaccines, due to vaccine pressure. While there is no evidence of a serovar drift in porcine pleuropneumonia, it has been suggested as a cause of vaccine failures in the related organism, Haemophilus paragallinarum. ${ }^{18}$ Hence, it is important that serotyping studies of the type reported here continue to be performed. Apparent instances of 
vaccine failures should certainly be investigated carefully, possibly using intensive surveys that involve the isolation and serotyping of multiple isolates of A pleuropneumoniae from such problem farms or herds.

\section{Acknowledgments}

The financial support of the Pig Research and Development Corporation is gratefully acknowledged. The assistance of our colleagues at the Animal Research Institute (Yeerongpilly), Toowoomba Veterinary Laboratory, the Elizabeth Macarthur Agriculture Institute (Camden), the Orange Regional Veterinary Laboratory, the Wollongbar Regional Veterinary Laboratory, the Bendigo Regional Veterinary Laboratory, the Central Veterinary Laboratory (Adelaide), the Animal Health Laboratory (Perth) and Murdoch University (Perth) in providing the field isolates used in this study is gratefully acknowledged. The collaboration of Dr R Nielsen (State Veterinary Serum Laboratory, Copenhagen, Denmark) who allowed Dr Blackall to work in her laboratory, is also gratefully acknowledged.

\section{References}

1. Nicolet J. Actinobacillus pleuropneumoniae. In: Leman AD, Straw BE, Mengeling WL, D'Allaire S, Taylor DJ, editors. Diseases of swine. lowa State University Press, Ames, 1992:401-408.

2. Nielsen R. Pleuropneumonia of swine caused by Haemophilus parahaemolyticus. Studies on the protection obtained by vaccination. Nord Vet Med 1976;28:337-348.

3. Nielsen R. Haemophilus pleuropneumoniae serotypes - cross protection experiments. Nord Vet Med 1984;36:221-234.

4. Mittal KR, Bourdon S, Berrouard M. Evaluation of counterimmunoelectrophoresis for serotyping Actinobacillus pleuropneumoniae isolates and detection of type-specific antigens in lungs of infected pigs. J Clin Microbiol 1993;31:2339-2342.
5. Eaves LE, Blackall PJ. Serological characterisation of Australian isolates of Actinobacillus pleuropneumoniae. Aust Vet J 1988;65:379-381.

6. Mittal KR, Higgins R, Larivière $S$. Determination of antigenic specificity and relationship among Haemophilus pleuropneumoniae serotypes by an indirect haemagglutination test. J Clin Microbiol 1983;17:787-790.

7. Mittal KR, Higgins R, Larivière $S$. Evaluation of slide agglutination and ring precipitation tests for capsular serotyping of Haemophilus pleuropneumoniae. $J$ Clin Microbiol 1982;15:1019-1023.

8. Mittal KR, Higgins $R$, Larivière $S$. Identification and serotyping of Haemophilus pleuropneumoniae by coagglutination. J Clin Microbiol 1983;18:1351-1354.

9. Mittal KR, Higgins R, Larivière S. Actinobacillus (Haemophilus) pleuropneumoniae serotype-8 isolates and their antigenic relationships with other $\mathrm{A}$ pleuropneumoniae serotypes. Am J Vet Res 1989;50:259-262.

10. Mittal KR. Cross-reactions between Actinobacillus (Haemophilus) pleuropneumoniae strains of serotypes 1 and 9. J Clin Microbiol 1990;28:535-539.

11. Blackall PJ, Pahoff J. Characterisation of porcine haemophili isolated from Australian pigs, 1988-1992. Aust Vet J 1995;72:18-21.

12. Gutierrez CB, Tascon RI, Vazquez JA, Ferri EFR. Cross-reactivity between Actinobacillus pleuropneumoniae serotypes comparing different antigens and serological tests. Res Vet Sci 1991;50:308-310.

13. Mittal KR, Bourdon S. Cross-reactivity and antigenic heterogeneity among Actinobacillus pleuropneumoniae strains of serotype 4 and 7. J Clin Microbiol 1991;29:1344-1347.

14. Reid GG, Blackall PJ. Comparison of adjuvants for an inactivated infectious coryza vaccine. Avian Dis 1987;31:59-63.

15. Mittal KR, Higgins R, Larivière $S$. Quantitation of serotype-specific and cross-reacting group-specific antigens by coagglutination and immunodiffusion tests for differentiating Actinobacillus (Haemophilus) pleuropneumoniae strains belonging to cross-reacting serotypes 3, 6, and 8. J Clin Microbiol 1988;26:985989.

16. Mittal KR, Higgins R, Larivière S. Serological studies of Actinobacillus (Haemophilus) pleuropneumoniae strains of serotype 6 and their antigenic relationship with other serotypes. Vet Rec 1988;122:199-203.

17. Alambedji RB, Dubreuil JD. Comparaison de deux antigènes de nature polysaccharidique pour le diagnostic sérologique par ELISA de la pleuropneumonie porcine (sérotype 5). J Vet Med B 1993;40:253-260.

18. Bragg RR, Coetzee L, Verschoor JA. Changes in the incidences of the different serovars of Haemophilus paragallinarum in South Africa: a possible explanation for vaccination failure. Onderstepoort J Vet Res 1996;63:217-226.

(Accepted for publication 9 October 1998)c

\section{Spermatozoa under peril?}

According to numerous studies, human sperm counts are diminishing. The trend has not been uniform and retrospective studies may suffer from a number or confounding factors, giving rise to debate over the validity of the decline and its possible causes.

DM de Kretser from Murdoch University has reviewed the current status of the controversy. Despite reservations about the methodology used to assess sperm counts, it is clear that there have been significant regional declines, unforeseen before this decade. In addition to diminishing sperm numbers, regional increases in the incidence of testicular cancer and cryptorchidism have been noted. It is not known whether these disorders share causality.

The development of Sertoli cells, key players in spermatogenesis, can be disturbed during pregnancy and puberty, leading to smaller sperm numbers later in life. The factors affecting Sertoli cells include chemicals and high ambient temperature. Oestrogenic substances, increasingly present in the environment, have been implicated. They include oral contraceptives, growth promoters, phyto-oestrogens, polychlorinated biphenyls, phenolic compounds and organochorine pesticides. High testicular temperature caused by tight underwear may be a factor of some significance.

Male human reproduction is vulnerable to adverse factors because of our long period of sexual immaturity and subsequent sexual activity. The effects may manifest after a prolonged exposure. While the decline in sperm numbers does not yet threaten male fertility, the author emphasises that it is vital for human reproductive health that the decline and its causes are investigated.

de Kretser DM. Are sperm counts really falling? Reprod Fertil Dev 1998;10:93-95. 